\title{
Solvent-free Cleavage of tert-Butyl Esters under Microwave Conditions
}

\author{
Doo Han Park $k^{*}$ and Jung Hwan Park \\ Department of Chemistry, Sahmvook Unwersin, Seoul 139-742. Korea. ${ }^{\circ}$ E-mail: dhparkosyuac.kr \\ Received fugtust 30, 2008, Accepted November 7, 2008
}

Key Words: tert-Butyl ester. Deprotection. Silica gel. Solvent-free. Microwave irradiation

Protection and deprotection of carbosylic acid with sterically bulky alkyl groups are essential steps in synthetic organic chemistry. Among them. tert-butyl is the most commonly used to protect the carboxylic acid group because the resulting tert-butyl esters are highly stable under neutral and basic conditions. and can be easily prepared from a variety of free carboxylic acids. The tert-butyl group could be removed usually by using strong acids such as $\mathrm{CF}_{3} \mathrm{COOH}{ }^{2} \mathrm{HNO}_{3}{ }^{3}$ and $\mathrm{H}_{2} \mathrm{SO}_{4}{ }_{4}$. However. major drawbacks of these strong acid conditions are formation of unwanted side products and decomposition of acid labile substrates. On the other hand, the use of Lewis acids such as $\mathrm{MgI}_{2}{ }^{5}$ and $\mathrm{ZnBr}_{2}{ }^{6}$ have a merit of mild reaction condition but need to long reaction time. higher reaction temperature, and involve toxic metals which are detrimental to the environment. In 2001 . Jackson reported an interesting method using silica gel as an efficient reagent for the cleavage of tert-butyl esters. Unfortunately this method also required long reaction time at high reaction temperature as exemplified by cleavage of tert-butyl from the tert-butyl t-methoxybenzoate took $7 \mathrm{~h}$ at reflux.

In recent years, microwave (MW) irradiation technique has been attracted much attention as a powerful method for the various organic transformations. ${ }^{8}$ Microwave heating has advantages of short reaction time. high chemical yield. clean reaction with easy work up. and solvent-free reaction condition." Although various kinds of reactions have been conducted under microwave irradiations to our best knowledge. only one example of deprotection of tert-butyl esters by using $p$-toluenesulfonic acid monohydrate under microwave condition has been published."

In this paper, we describe the simple and effective method for the cleavage of tert-butyl esters by combination of silica gel (230-400 mesh, standard grade for flash column chromatography) and two different type of microwave irradiation (CEM Discover microwave as a single-mode microwave unit and CEM MARS5 microwave as a multi-mode microwave unit) in solvent-free conditions (Scheme 1).

The reactions were carried out by simple mixing tert-butyl esters with silica gel followed by irradiation with a temper-

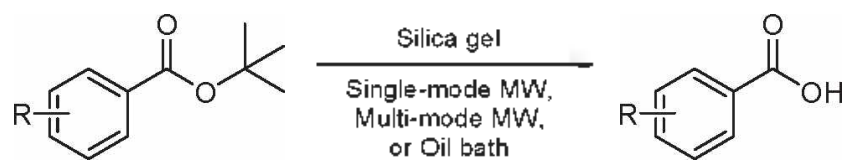

Scheme 1. Solvent-free cleavage of tert-butyl group under microwave iradiations ature controlled single-mode or multi-mode microwave reactor. For comparisons, the same reaction has been carried out under conventional heating condition. After screening the reaction temperature. we found that irradiation of tert-butyl benzoate doped into silica gel at $120^{\circ} \mathrm{C}$ for $3.5 \mathrm{~min}$ using single-mode MW showed complete cleavage of the tert-butyl group resulted in benzoic acid in $>99 \%$ yield (Entry 1. Table 1). Utilization of multi-mode $\mathrm{MW}$ required slightly longer reaction time, thus, the reaction was completed in 5 min irradiation. In contrast. when the same reaction was conducted under conventional thermal condition at $120^{\circ} \mathrm{C}$. it took $3 \mathrm{~h}$ reaction time to obtain benzoic aid in $90 \%$ yield. Various tert-butyl esters have been investigated. and the results are summarized in Table 1. As shown in Table 1, all of the tert-butyl esters examined can be cleavage effectively under MW condition with strikingly shorted reaction time ( $3-5 \mathrm{~min})$ compare to that required under conventional heating condition. Moreover, the MW irradiation increased chemical vield. It has been also found that single-mode MW irradiation is slightly more effective than the multi-mode MW irradiation, which could ascribe to the focused irradiation increasing local nicro-heating. The electron density in aromatic ring did not much affect the efficiency of the present method.

In conclusion we have developed a sintele and effective method for the cleavage of tert-butyl esters using silica gel in solvent-free condition under microwave irradiation. This method is remarkable for high chemical yield and the operational simplicity of the procedure is also attractive.

\section{Experimental Section}

Microwave irradiation was carried out with CEM Discover microwave at 30 watts as a single-mode microwave unit and CEM MARS5 microwave. at 600 watts as a multi-mode microwave unit. ${ }^{1} \mathrm{H}$ NMR spectra were obtained on a BRUKER 300 in DMSO- $d_{6}$ with TMS as an internal standard.

General procedure for the cleavage of tert-butyl esters: tert-Butyl ester $(10 \mathrm{mmol})$ doped on a silica gel $(2.5 \mathrm{~g})$ was mixed thoroughly' on a vortex mixer. The reaction mixture was subjected to single-mode microwave at 30 watts for $3.0-3.5$ min. or multi-mode microwave at 600 watts for $4.5-5.0 \mathrm{~min}$. or conventional heating use of oil bath at $120^{\circ} \mathrm{C}$ for $180 \mathrm{~min}$. Upon conpletion of the reaction, the reaction mixture was diluted with $10 \%$ methanol in dichloromethane. and the reaction nuxture was filtered through a pad of Celite ${ }^{\circledR}$. Finally, the filtrate was evaporated under reduced pressure to afford the product. 
Table 1. Cleavage of tert-butyl esters with silica gel in solvent-free conditions

\begin{tabular}{|c|c|c|c|c|c|c|c|c|c|c|}
\hline \multirow[b]{2}{*}{ Entry } & \multirow[b]{2}{*}{ Ester } & \multicolumn{3}{|c|}{ Single-mode MW $(30 \mathrm{~W})$} & \multicolumn{3}{|c|}{ Multimode MW (600 W) } & \multicolumn{3}{|c|}{ Conventional heating (oil bath) } \\
\hline & & $\begin{array}{l}\text { Time } \\
\text { (minic) }\end{array}$ & $\begin{array}{c}\text { Temp. } \\
\text { (C) }\end{array}$ & $\begin{array}{l}\text { Yield } \\
(\%)^{a}\end{array}$ & $\begin{array}{l}\text { Time } \\
\text { (min.) }\end{array}$ & $\begin{array}{l}\text { Temp. } \\
\text { (C) }\end{array}$ & $\begin{array}{l}\text { Yield } \\
(\%)^{\circ}\end{array}$ & $\begin{array}{l}\text { Time } \\
\text { (min.) }\end{array}$ & $\underset{\left({ }^{\circ} \mathrm{C}\right)}{\text { Temp. }}$ & $\begin{array}{l}\text { Yield } \\
(\%)^{a}\end{array}$ \\
\hline 1 & & 3.5 & 120 & 99 & 50 & 120 & 99 & 180 & 120 & 90 \\
\hline 2 & & 3.5 & 120 & 99 & 50 & 120 & 98 & 180 & 120 & 92 \\
\hline 3 & & 3.5 & 120 & 99 & 4.5 & 120 & 99 & 180 & 120 & 92 \\
\hline 4 & & 3.5 & 120 & 98 & 5.0 & 120 & 98 & 180 & 120 & 89 \\
\hline 5 & & 3.0 & 120 & 99 & 5.0 & 120 & 99 & 180 & 120 & 88 \\
\hline 6 & & 3.0 & 120 & 99 & 50 & 120 & 99 & 180 & 120 & 91 \\
\hline 7 & & 3.5 & 120 & 97 & 5.0 & 120 & 95 & 180 & 120 & 82 \\
\hline 8 & & 3.5 & 120 & 97 & 5.0 & 120 & 94 & 180 & 120 & 84 \\
\hline 9 & & 3.5 & 120 & 95 & 5.0 & 120 & 91 & 180 & 120 & 78 \\
\hline 10 & & 3.5 & 120 & 99 & 5.0 & 120 & 99 & 180 & 120 & 90 \\
\hline
\end{tabular}

${ }^{a}$ Isolated yield.

Benzoic acid (Entry 1): ô $7.48(\mathrm{t}, J=7.56 \mathrm{~Hz} .2 \mathrm{H} .2 \times \mathrm{CH})$. $7.61(\mathrm{t} . J=7.43 \mathrm{~Hz}, \mathrm{lH} . \mathrm{CH}) .8 .1 \mathrm{l}$ (d. $J=7.12 \mathrm{~Hz}, 2 \mathrm{H} .2 \times \mathrm{CH}$ ). 4-Methoxybenzoic acid (Entry 2): $\delta 3.86\left(5,3 \mathrm{H} . \mathrm{OCH}_{3}\right)$. $6.92(\mathrm{~d}, J=8.86 \mathrm{~Hz}, 2 \mathrm{H}, 2 \times \mathrm{CH}) .8 .03$ (d $J=8.85 \mathrm{~Hz}, 2 \mathrm{H}, 2 \times \mathrm{CH}$ ).

2-Methylbenzoic acid (Entry 3): $\hat{o} 2.67$ (s. $3 \mathrm{H} . \mathrm{CH}_{3}$ ). $7.26-7.31$ (m. $2 \mathrm{H} .2 \times \mathrm{CH}) .7 .46$ (t. $J=7.46 \mathrm{~Hz}, 1 \mathrm{H} . \mathrm{CH}), 8.08$ (d. $J=8.42 \mathrm{~Hz}, \mathrm{IH} . \mathrm{CH}$ ).

3-Methylbenzoic acid (Entry 4): ò $2.42\left(\mathrm{~s}, 3 \mathrm{H} . \mathrm{CH}_{3}\right) .7 .39$ (t. $J=15.60 \mathrm{~Hz}, 1 \mathrm{H} . \mathrm{CH}) .7 .39$ (d. $J=15.70 \mathrm{~Hz}, 1 \mathrm{H} . \mathrm{CH}) .7 .93$ (d. $J=7.10 \mathrm{~Hz} .1 \mathrm{H} . \mathrm{CH}), 7.94(5,1 \mathrm{H}, \mathrm{CH})$.

4-Methylbenzoic acid (Entry 5): $\delta 2.44(\mathrm{~s}, 3 \mathrm{H}), 7.28(\mathrm{~d} . J=$ $8.07 \mathrm{~Hz}, 2 \mathrm{H} .2 \times \mathrm{CH}$ ). 8.01 (d. $J=8.19 \mathrm{~Hz}, 2 \mathrm{H} .2 \times \mathrm{CH}$ ).

4-Bmmobenzoic acid (Entry 6): $\delta 7.57(\mathrm{~d}, J=8.39 \mathrm{~Hz}, 2 \mathrm{H}$, $2 \times \mathrm{CH}) .7 .92(\mathrm{~d}, J=8.40 \mathrm{~Hz}, 2 \mathrm{H}, 2 \times \mathrm{CH})$.
4-Trifluoromethỵlbenzoic acid (Entry 7): $\delta 7.70(\mathrm{~d} . J=8.18$ Hz. $2 \mathrm{H}, 2 \times \mathrm{CH}), 8.18(\mathrm{~d}, J=8.12 \mathrm{~Hz}, 2 \mathrm{H}, 2 \times \mathrm{CH})$

4-Nitrobenzoic acid (Entry 8): $\delta 8.15$ (d. $J=11.13 \mathrm{~Hz} .2 \mathrm{H}$, $2 \times \mathrm{CH}) .8 .32(\mathrm{~d}, J=11.14 \mathrm{~Hz}, 2 \mathrm{H}, 2 \times \mathrm{CH})$

Nicotinic acid (Entry 9): $\delta 7.55$ (d. $J=4.83 \mathrm{~Hz}, 1 \mathrm{H}, \mathrm{CH}$ ). $8.28($ t. $J=1.95 \mathrm{~Hz}, 1 \mathrm{H} . \mathrm{CH}) .8 .79$ (d. $J=1.68 \mathrm{~Hz} .1 \mathrm{H}, \mathrm{CH}$ ), 9.07 (s. lH. CH).

2-Furoic acid (Entry 10): $\delta 6.64$ (dd, $J=1.53 \mathrm{~Hz} \& 1.74 \mathrm{~Hz}$, lH. CH). $7.20(\mathrm{~d}, J=3.47 \mathrm{~Hz}, \mathrm{lH}, \mathrm{CH}) .7 .90(\mathrm{~s} . \mathrm{H}, \mathrm{CH}$ ).

Aclanowledgments. This work was supported by the SalumYook University Research Fund in 2008. We also thank Dr. Jae Kyun Lee (Korea Institute of Science and Technology) for his technical assistance. 


\section{References}

1. Green, T. W: Wuts, P. G. Protective Groups in Organic Synthesis, $4^{\text {th }}$ ed; Toln Wiley \& Sons: New York, 2007. (b) Kocienski, P. I. Protecting Groups, $3^{\text {rd }}$ ed. George Thieme Stuttuart: New York, 2004.

2. Bryan, D. B.; Hall, R. F.: Holden, K. G.: Huffnan, W. F.: Gleason, J. G. J. Am. Chem. Soc: 1977, 99, 2353.

3. (a) Strazzolini, P.; Dall Arche, M. G.; Giumanini, A. G. Tetrahedron Lett. 1998, 39, 9255. (b) Strazzolini, P.; Scuccato, M.; Giuanini, A. G. Tetrahedron 2000, 56, 3625 .

4. Strazzolini, P.: Misuri, N.: Polese, P. Tetrahedron Lett. 2005, 16 , 2075

5. Martinez, A. G.; Barcina, T. O.; Veccio, G. H. d.: Hanack, M.; Subramanian, L. R. Tetrahedron Lett. 1991, 32, 5931.
6. (a) Wu, Y.q.; Limburg, D. C.; Wilkinson, D. E.; Vaal, M. T.; Hamilton, G. S. Tetrahedron Lett. 2000, H1, 2847. (b) Kaul, R: Broulillette, Y.: Saijadi, Z.: Hanstord, K. A.: Lubell, W. D. J. Ong. Chem. 2004, 69,6131.

7. Tackson. R. W. Tetrahedron Lett. 2001, 42,5163.

8. (a) Caddick, S. Tetrahedron 1995, 51, 10403. (b) Loupy, A. ificrowave in Oiganic Symhesis: Wiley-VCH: Weinheim, 2002.

9. (a) Vama, R. S. Pute and Applied Chentistry 2001, 73, 193. (b) Park, S. H. Bull. Korean Chent. Soc 2003, 24, 253. (c) Park, S. H.; Gwon, H. T.; Lee, H. S.: Park, K. B. Bull Korean Chem Soc. 2005, 26, 1701. (d) Io, E. A.: Aln, J. A.: Jun, C. H. Bull Korean Chem. Soc 2007, 28, 2020. (e) Yadav, T. S.: Reddy, B. V. S.; Shankar, K. S.; Swamy, T.; Premalatha, K. Bull. Kowem Chem. Soc. $2008,29,1418$.

10. Lee, J. C.; Yoo, E. S.: Lee, I. S. Swnth. Comm $2004,34,3017$. 